\title{
Imaging Informatics Finally Breaks into the Enterprise
}

\author{
James Whitfill ${ }^{1,2}$ (1)
}

Published online: 25 August 2016

(C) Society for Imaging Informatics in Medicine 2016

In an era when the US health system is wrestling with the consequences of the rapid and wrenching adoption of electronic healthcare records, it is worth pointing out that the first informatics revolution of the modern era in healthcare began in the 1990s with the adoption of PACS systems to replace film with digital image-based systems. This revolution not only drove down the cost of care but also improved physician productivity: two goals we have yet to see from mainstream EHRs. Yet, over the past several years, a new movement within imaging informatics has quietly been taking place: the expansion of digital imaging outside of radiology and into other clinical departments. Ironically, it was the adoption of the EHR which has provided the framework for Enterprise Imaging to gain more mainstream acceptance. The ability of the EMR to host an embedded viewer allows other departments to realize that the images they acquire can bring value to a variety of clinicians who practice within a collaborative space. At the same time, in an era of HIPAA security and increasing budgetary pressures to save on storage costs, IT leaders see the chance to provide best practice security and storage practices to disparate image pools. These forces have come together to drive the next wave in imaging informatics: Enterprise Imaging. This wave brings the promise of skin lesions, retinal photos, endoscopy views, and digitized pathology slides all visible in the same viewer embedded

James Whitfill

jwhitfill@shpcare.com

1 Innovation Care Partners, 8901 E Mountain View, Suite 130, Scottsdale, AZ 85258, USA

2 Departments of Internal Medicine and Biomedical Informatics, University of Arizona College of Medicine-Phoenix, Phoenix, AZ, USA within the patient's electronic chart. In this edition of the Journal of Digital Imaging, the HIMSS-SIIM Enterprise Imaging workgroup has produced a series of white papers which capture the current state of Enterprise Imaging, lay out the current path for adoption, and set the path for the future.

In the 1990s, organizations like the Society for Computer Applications in Radiology created a collaborative space for early adopters who were attempting to assemble and optimize early PACS systems. As the first sites driving towards Enterprise Imaging sought a new home for the same kind of collaboration, it was natural for HIMSS and SIIM to collaborate to form a workgroup to fill that need. Over the past 24 years, healthcare informatics has matured as a field to include the entire enterprise, especially with the rise of EHRs. It therefore makes sense that in order to coalesce into a formal discipline, Enterprise Imaging needed the support of both an organization focused on the science of imaging informatics as well as one focused on the best use of information technology in the USA and Canada.

Authors from a variety of organizations including academic institutions, community hospitals, and vendors have worked to create practical white papers that allow novice users to readily understand the space of Enterprise Imaging while more sophisticated users will appreciate the examples of best practices and standards utilization. One of the key issues for Enterprise Imaging is the challenge to balance experience gained from its radiology roots without forcing a radiology centric workflow across disparate clinical workflows. The nurse caring for a victim of spousal abuse in the ED, the dermatologist in her clinic, and the surgeon in the OR each see the value of sharing their clinical images as part of the medical record but cannot interrupt their care to create an order-based workflow that makes no sense to their clinical practice. Dawn Cram, Chris Roth, and Alexander Tobin nicely 
tease apart this challenge in their paper of "Orders Versus Encounters Based Image Capture." In a parallel manner, the workgroup itself has had to create an openness to accommodate best clinical workflow within different departments that does not forget the importance of image integrity.

Amy Vreeland et al. have created a framework with specific use cases to illustrate the clinical reasons for and the technical standards used within cross enterprise sharing. By walking through the roles that DICOM and XDS play in this process, the authors set up the blueprint for true image sharing. They then perform a gap analysis to identify the myriad shortcomings still faced in practice today which in turn allows this field to focus on new solutions to these gaps. Finally, in "The Current State and Path Forward for Enterprise Image Viewing," Roth, Lannum, and Tobin enumerate the characteristics of an enterprise viewer and draw out the distinctions with a departmental viewer like a traditional PACS.

These papers along with the others herein provide the foundation of a new and rapidly evolving space in Medical Informatics. The authors and the entire HIMSS-SIIM workgroup should be congratulated for their hard work in shaping this new discipline and producing content that can now allow other institutions and vendors to accelerate the development of new advances in this space. After so many years of struggle with Meaningful Use, the dawn of Enterprise Imaging is a welcome development within healthcare informatics. Importantly, it is one which should bring immediate and tangible benefits to the clinicians caring for patients. 